\title{
Collective Background Extraction for Station Market Area by Using Location Based Social Network
}

\author{
Kousuke Kikuchi ${ }^{1}$, Tatsuto Kihara ${ }^{2}$, Atsushi Enta ${ }^{3}$, Hideaki Takayanagi ${ }^{2}$, Takeshi Kimura ${ }^{4}$, Kazuto Hayashida ${ }^{1}$ \\ and Hitoshi Watanabe ${ }^{1}$ \\ 1. Department of Creative Science and Engineering, Waseda University, Tokyo 169-8555, Japan \\ 2. School of Environmental Science, University of Shiga Prefecture, Shiga 522-8533, Japan \\ 3. Faculty of Science and Technology, Tokyo University of Science, Tokyo 121-0062, Japan \\ 4. A\&A Co. Ltd., Tokyo 101-0062, Japan
}

\begin{abstract}
Half centuries of follow-up survey has enabled the architects and urban planners to design rationally by the aid of planning. Nonetheless, limitation has occurred at planning because city has been changing its utility in accordance with its users' demand. In this paper, the authors proposed a method to analyze trait of users in market areas near stations by analyzing location based social network. After the datum collection from geotagged tweets, these GPS (global positioning system) datum were plotted to map attained from yahoo open location platform. Then the morphological analysis and terminology extraction system extracted the keywords and their scores. After calculating the distance from stations and users' GPS coordination, the authors extracted the array of keywords and corresponding scores in some station market area. Lastly, ratios of all users' scores and city's scores were calculated to examine the locality. Full combination of data collection, natural language processing and visualization enabled the authors to envisage distribution of collective background in city.
\end{abstract}

Key words: Location based social networks, natural language processing, market analysis, visualization, big data.

\section{Introduction}

\subsection{Research Methods on City}

For over few decades, the quantitative or qualitative methods to analyze market area have been studied not only to fathom the potential of market, but also to study the characteristics of indigenous location. For example, transition probability model was proposed by Nakamura to extrapolate human distribution in certain city [1]. Also, in the Great Britain, Hiller introduced Space Syntax based on the graph theory and pedestrian's recognition of city [2]. These quantitative and mathematical methods have bolstered the background of architectural and urban planning. On the other hand in qualitative analysis, follow-up

Corresponding author: Kousuke Kikuchi, M.Arch., Ph.D. student, research fields: urban study, architectural planning, social computing, visualization, sensoring and digital fabrication. E-mail: kousukekikuchi@toki.waseda.jp. surveys were rendered to study indigenous traits of city's user. For instance, Lynch clarified that human cognition was basically consisted of five elements [3]. In addition, Hayden proposed Genius Loci, utilizing oral history of native inhabitants [4]. These attempts analyzed the qualitative aspects of a city.

\subsection{Problems on the Research Methods}

Although these methods have been utilized at planning and designing, the limitations have been occurred in both methods. Among quantitative methods, one of the major problems is that the parameter of the methods ignores the diversity of recent user. Due to information technology, the authors' behavior got purpose-oriented. For example, by using search engine such as Google, pedestrians can have the destination beforehand. As a result, information about purpose or function to arrive at city can be added to people' destination determination 
process as a parameter.

On the other hand, among qualitative methods, one of the major problems is that follow-up survey might change behavior of the targeted persons. Since researcher must follow the person, the distorted atmosphere of the research can be different from that of usual one and emerged stress which culminates the altering behavior. Indeed, the behavior of the person is basically deviated from the usual in the qualitative method.

Lastly, these methods are thought to never predict the changing utility of a city. In fact, strong and potential demand of people can alter the function or stores of area. As modern economy has shown the importance of human factor, collective personalities in come area can be with great significance to predict its other possibility of function.

\subsection{Location Based Social Networks}

To solve the problems, the authors proposed to utilize a location based social network, Twitter. Firstly, one of the benefits is quantity of the data. Recent information technology has developed social networks with GPS (global positioning system) data. Using the location based social network as a platform of users' data enable researchers to obtain googolplex of data. Secondly, being different from qualitative method, social media will not change the behavior of its user. Hence, data of social media has more reality than follow-up survey. Finally, other benefit is that by using big data of each user, the authors can analyze the trait of user as well as location being same as qualitative method. Indeed, using location based social networks enable them to sublate quality and quantity of data.

\subsection{Premises and Hypothesis toward a New Method}

The authors presume two premises based on the Florida's assertion [5]. Florida's view toward city can be consisted of accumulation of each person living in a city. The authors consulted on this insistence to make premises and hypothesis. One premise is that user's traits can be presented at frequency of the words. After walking on the snow, people' footprints can be seen. In other word, people' personalities emboss into city. Another premise is that sum of the frequency of the word in a city expresses the characteristics of city. Also, high ratio between the characteristics in city and all users' characteristics will determine the potential of city.

\subsection{Purpose of This Paper}

This paper fabricates the system to extrapolate the market characteristics by assessing the users of Twitter and finds other potential function in a city.

\section{Data Collection and Visualization}

Fig. 1 illustrates the procedures of data collection and visualization of the GPS dots which can be obtained via Twitter.

\subsection{Data Collection}

The authors made two programs to list the geo-tagging users and to obtain their tweets. One program collected the geo-tagging user list by using public streams, extracting only the geo-tagging user near Japan [6]. The other program obtained the each user's tweets based on user timeline of API (application programming interface) and outputted the each user's username, time, tweet and GPS coordination into CSV (comma-separated values) format file [7]. Two years of executing these programs were shown in Table 1.

\subsection{Visualization}

The authors made a program which visualized GPS data plotting into map. The authors employed YOPL (yahoo open location platform) static map to get map [8]. Traits of YOPL are Mercator projection and zoom level. One zoom level represents entire whole earth in $256 \times 256$ pixels. As the zoom level increase by one, the magnitude of map becomes doubled. To plot the information, the authors implemented the coordination 


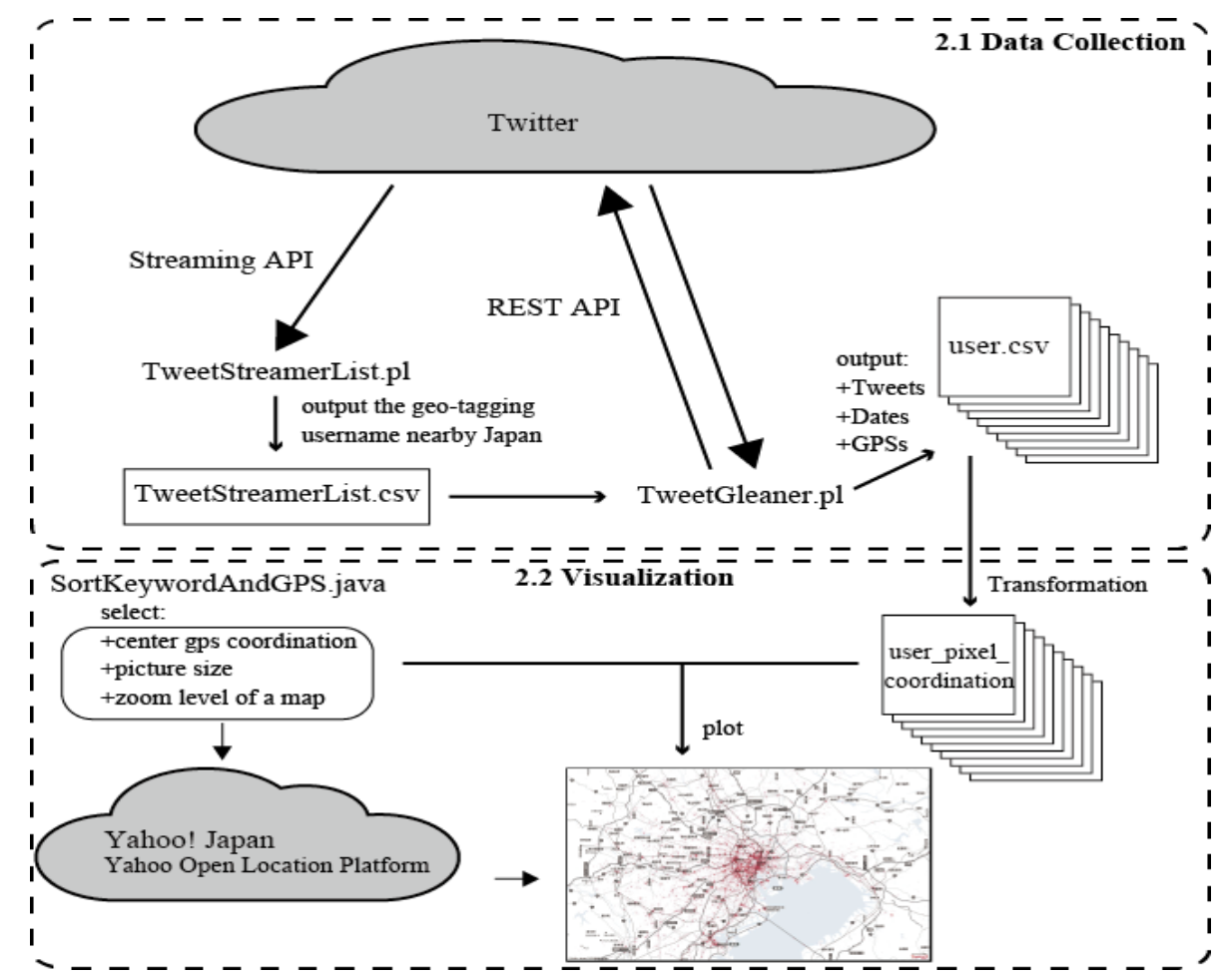

Fig. 1 Data collection and visualization.

Table 1 Data collection summary.

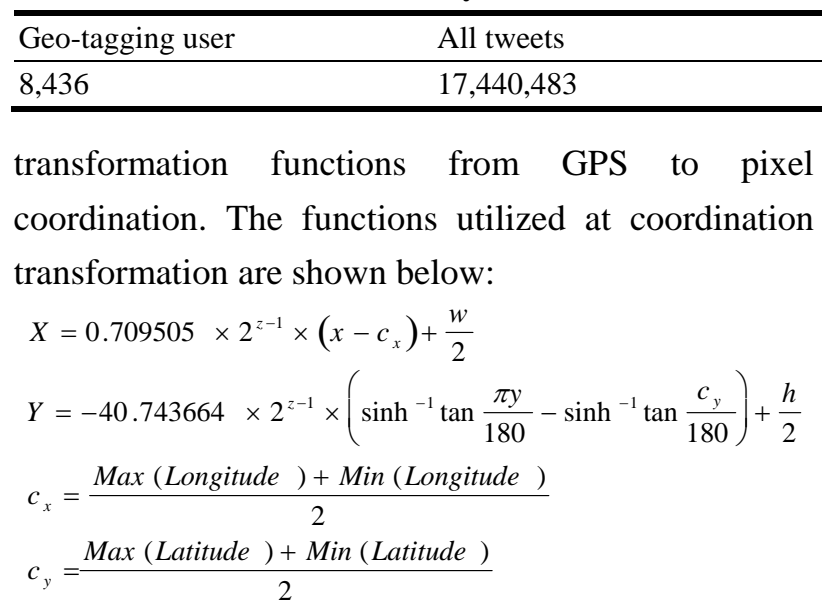

$X$ : pixel coordination converted from longitude;

$Y$ : pixel coordination converted from latitude;

$z$ : zoom level;

$x$ : longitude from GPS data;

$y$ : latitude from GPS data;

$c_{x}$ : center of the longitude;

$c_{y}$ : center of the latitude.

The limitation of these functions arises from longitude. Since the center of the map is determined by the maximum and minimum of longitude and
All geo-tagged tweets $\quad$ Percentage

$2,380,704 \quad 0.136504476$

latitude, people in opposite side of Greenwich are not calculated the proper center of picture. Nevertheless, in this paper, subject of data was limited to Japan. Although the authors will ponder more genetic method, this method was applied.

\subsection{Location of Geo-tagged Tweets}

By plotting the GPS data into Tokyo area, the authors confirmed the traits of geo-tagged tweets. Fig. 2 illustrates the relationship between all geo-tagged tweets and the major stations in Tokyo. From the picture, geo-tagged tweets were distributed near the stations. In short, location based social network enables us to analyze the city with mass public transportation system.

\section{Market Analysis}

Fig. 3 represents the diagram of the procedures in this analysis. 


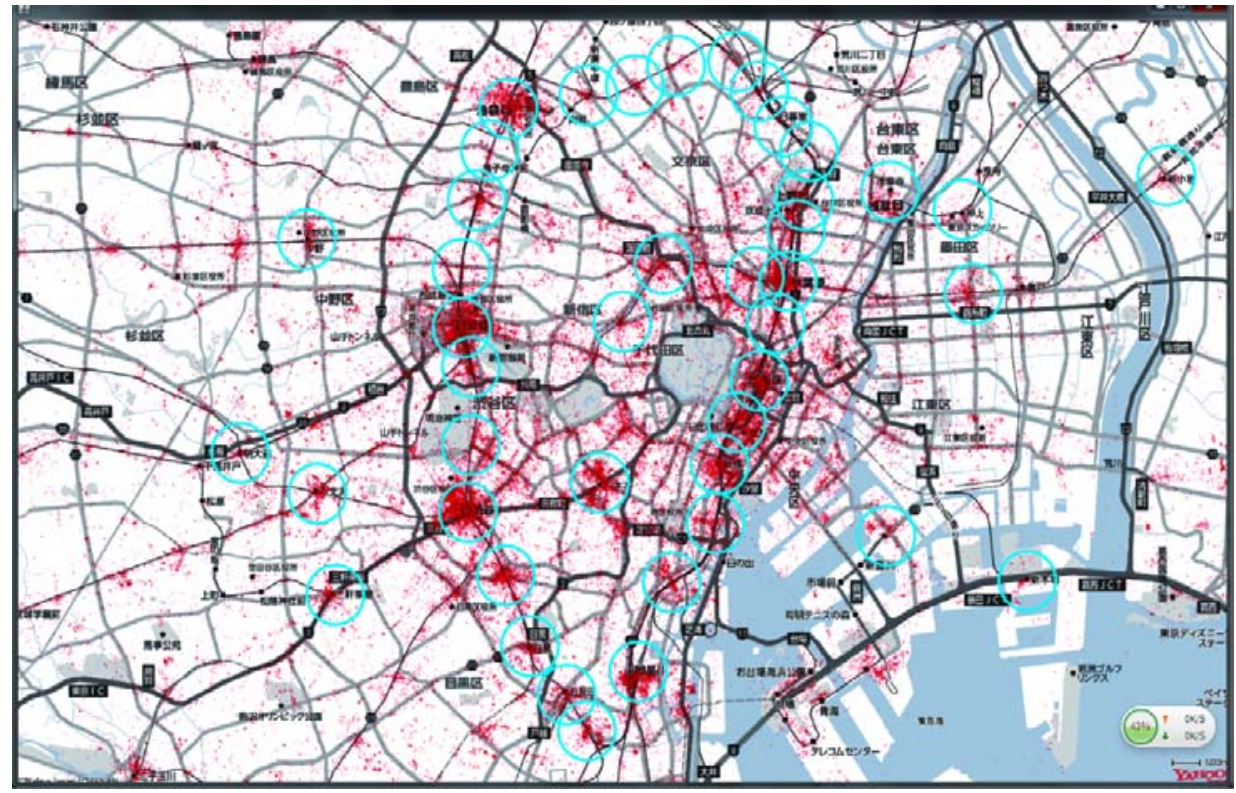

Fig. 2 Relationship between major stations in Tokyo and geo-tagged tweets.

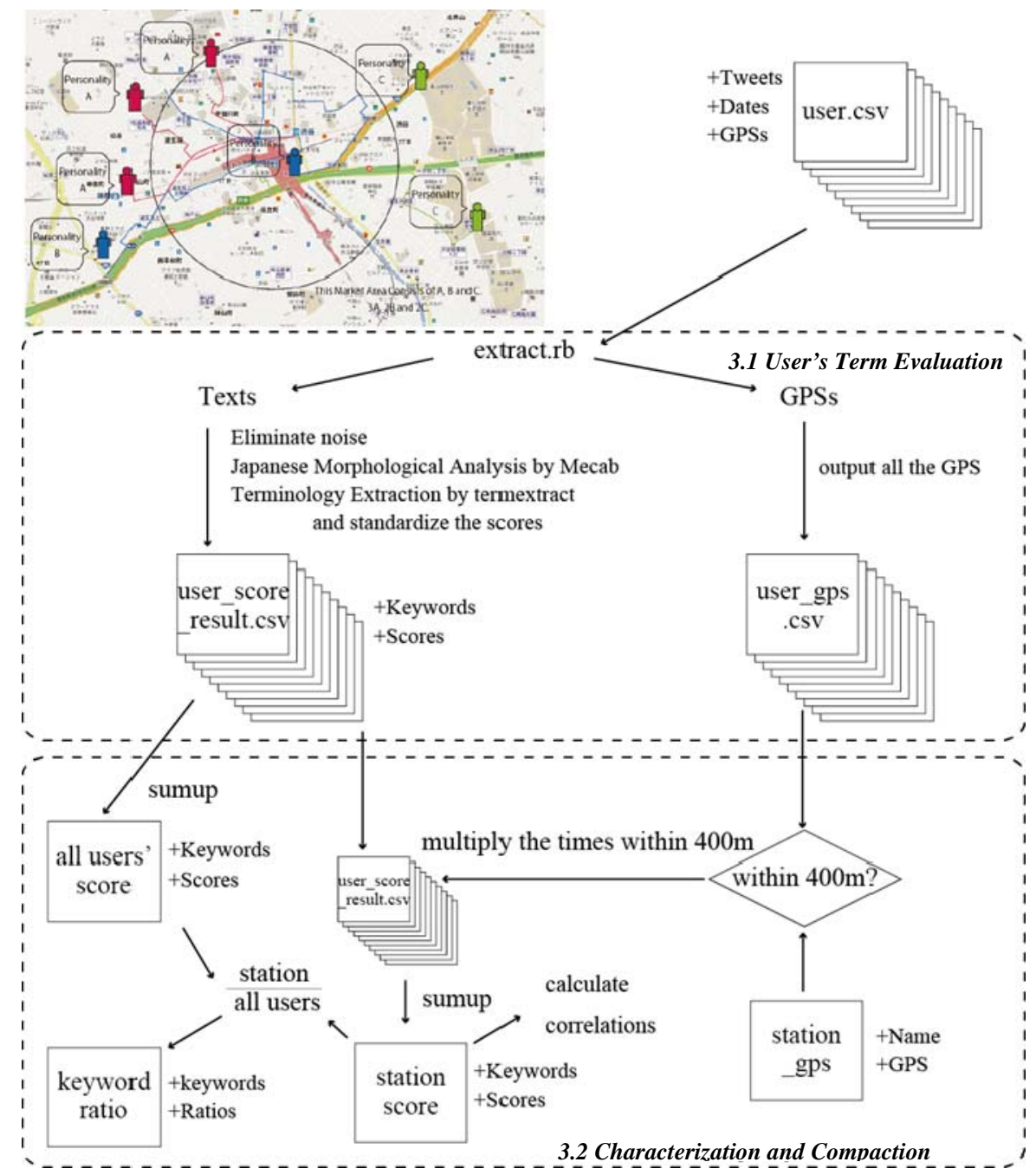

Fig. 3 Diagram of procedure for market evaluation. 


\subsection{User's Term Evaluation}

User analysis program consists of three steps: extraction of GPS and text, Japanese morphological analysis, term evaluation. At first, GPS coordination and text data were extracted from each user files, and GPS data was outputted. Noise information such as mark, smiley and colloquial expression was eliminated or replaced to proper expression. Next, Japanese Morphological Analyzer, MeCab, separated the words and estimated the part of speeches [9]. Lastly, Term Extract evaluated the important terms with scores [10]. Terminologies and their score were written down to each user's file.

\subsection{Characterization and Compaction}

Station GPS coordination was attained from the web service [11]. Stations near Tokyo were extracted by using GPS. Next, the authors made a program to count the numbers of GPS to enter the market area, within 400 meters from each station by using Hubeny Formula. The authors determined 400 meters because $80 \%$ of people can walk without any difficulty. Table 2 shows the numbers of geo-tagged tweets and station names.

All the scores of each user's keywords were multiplied the number entered market area. After that, the keywords and scores in market area were extracted by adding all the scores of each user. Also, for the comparison, the authors also extracted the whole users' keywords and equivalent scores. Finally, the authors calculate the ratios of scores dividing scores of city by whole users' score. As small percentage of score culminated relatively high ratio, the authors limited the keywords frequency over $1.0 \times 10^{-3}$.

\subsection{Analyzing Methods}

From Table 2, the authors extracted seven stations which have the largest number of GPS within station market areas: Shinjuku, Shibuya, Akihabara, Tokyo, Shinagawa, Roppongi and Ginza. Since Tokyo, Shibuya, Shinagawa and Shinjuku work as terminals, there are number of lines in these stations. Also, their areas have business districts and general commercial areas. On the other hand, Akihabara, Roppongi and Ginza are considered as contents-based-areas because there are unique traits in vicinity.

To examine the trends, two methods were devised: First method was calculating correlation among seven stations by using term and score; the other method is hypernym method. By generalizing the meaning of term, same categories were added to each terms' score.

\section{Results and Analysis}

\subsection{Correlations among Seven Stations}

First method was calculating correlation between seven stations by using term and score. The other method is generalizing the meaning of term and adding the scores. Correlations of seven stations were shown in Table 3.

Roppongi had significant difference between other stations since all the rates were below 0.20. According to this analyzing, some similarities can be found such

Table 2 Rank of number of GPS.

\begin{tabular}{|c|c|c|c|c|c|}
\hline No. & Station & Number of GPS & No. & Station & Number of GPS \\
\hline 1 & Shinjuku & 21,716 & 11 & Nihonbashi & 8,133 \\
\hline 2 & Shibuya & 21,532 & 12 & Suehirocho & 7,586 \\
\hline 3 & Akihabara & 19,505 & 13 & Ebisu & 7,502 \\
\hline 4 & Tokyo & 15,189 & 14 & Mitsukoshi Mae & 7,213 \\
\hline 5 & Shinagawa & 12,415 & 15 & Hibiya & 6,781 \\
\hline 6 & Roppongi & 11,695 & 16 & Tocho-Mae & 6,620 \\
\hline 7 & Ginza & 11,248 & 17 & Iwamotocho & 6,499 \\
\hline 8 & Shinbashi & 10,186 & 18 & Shinsen & 5,503 \\
\hline 9 & Yurakucho & 10,060 & 19 & Ueno & 5,459 \\
\hline 10 & Ikebukuro & 8,836 & 20 & Meiji Jingu Mae & 5,364 \\
\hline
\end{tabular}


Table 3 Correlation coefficients of each station.

\begin{tabular}{|c|c|c|c|c|c|c|c|}
\hline & Ginza & Akihabara & Shibuya & Shinjuku & Tokyo & Shinagawa & Roppongi \\
\hline Ginza & - & $0.301219^{b}$ & $0.265179^{b}$ & $0.381081^{b}$ & $0.43233^{c}$ & $0.350343^{b}$ & $0.139475^{a}$ \\
\hline Akihabara & $0.301219^{b}$ & - & $0.117386^{a}$ & $0.243911^{b}$ & $0.187813^{a}$ & $0.501648^{c}$ & $0.05169^{a}$ \\
\hline Shibuya & $0.265179^{b}$ & $0.117386^{\mathrm{a}}$ & - & $0.50956^{c}$ & $0.122547^{\mathrm{a}}$ & $0.16599^{a}$ & $0.141812^{a}$ \\
\hline Shinjuku & $0.381081^{b}$ & $0.243911^{b}$ & $0.50956^{\mathrm{c}}$ & - & $0.238245^{b}$ & $0.176039^{a}$ & 0.120964 \\
\hline Tokyo & $0.43233^{c}$ & $0.187813^{b}$ & $0.122547^{a}$ & $0.238245^{b}$ & - & $0.290291^{b}$ & $0.04048^{a}$ \\
\hline Shinagawa & $0.350343^{b}$ & $0.501648^{c}$ & $0.16599^{a}$ & $0.176039^{a}$ & $0.290291^{b}$ & - & $0.051094^{a}$ \\
\hline Roppongi & $0.139475^{\mathrm{a}}$ & $0.05169^{a}$ & $0.141812^{a}$ & $0.120964^{a}$ & $0.04048^{\mathrm{a}}$ & $0.051094^{\mathrm{a}}$ & - \\
\hline
\end{tabular}

${ }^{\mathrm{a}}$ Extreme weak correlation; ${ }^{\mathrm{b}}$ Weak correlation; ${ }^{\mathrm{c}}$ Middle correlation.

as Tokyo and Ginza, Shibuya and Shinjuku, Akihabara and Shinagawa.

\subsection{Frequently Used Words in Seven Stations}

The authors extracted the keywords with ratio above 2.0, tagged hypernym, a word more generic than a given word, and summed up the scores of hypernym. The results were shown in Table 4. As shown, the terms, related to "place" and "public transportation", were scored higher than other hypernym, since most geo-tagged tweets can be found near the stations. Among the seven stations, Tokyo has the most hypernym of "place" and "public transportation" because of the busiest terminals all over the world.

\subsection{Common Words in Terminal-Type Stations}

In Table 4, “commodity”, “meal” and “shop” were common hypernyms in terminal-type stations. Other common hypernyms were internet related term although the traits of cities were different from each city. In Shibuya, "IT (internet technology)” was high common hypernym and less “internet slang”. Shibuya has SNS based IT companies. In Shinagawa, high "IT" and less "Internet Slang” were found. Shinagawa has ordinal IT. Same level of "IT" and "internet slang”, "greeting" and "work" can be found in Tokyo. Near Tokyo Station, there are headquarters of Japanese companies. These differences of companies might culminate the difference of frequent use of hypernyms.

\subsection{Common Words in Contents-Type Stations}

Among contents-type stations, Roppongi and Akihabara had distinctive characters. In Roppongi, the hypernyms related to "sightseeing spots", "foreign countries”, “foreigners" and "sub-culture” were predominated. In fact, many foreign embassies are located and also night life is popular with locals and foreigners. However, "sub-culture" in Roppongi seems uncommon because few evidences has been shown that characteristics of Roppongi were consisted of sub-culture. This fact indicates that Roppongi may have the potential of sub-culture.

In Akihabara, “events”, “IT”, “sub-culture” and "internet slang” were common hypernyms since Akihabara is Mecca of sub-culture of Otaku. There were numbers of events on idles and games and also stores on idles, games, comics, PC parts and electronics. Hence, these hypernyms are worth reliable.

In Ginza, notable hypernym was "politics”. Of course, there is no major political party in Ginza. The fact seems to reflect the people in Ginza tend to have interest on politics.

\section{Discussion}

Some similarities and disparities can be found from correlation and hypernym methods. Firstly, relatively high correlations were due to the high ranks of keywords and scores. In Tokyo and Ginza, top three hypernyms were the same. Also in Shinagawa and Akihabara, place, IT, transportation, internet slang 


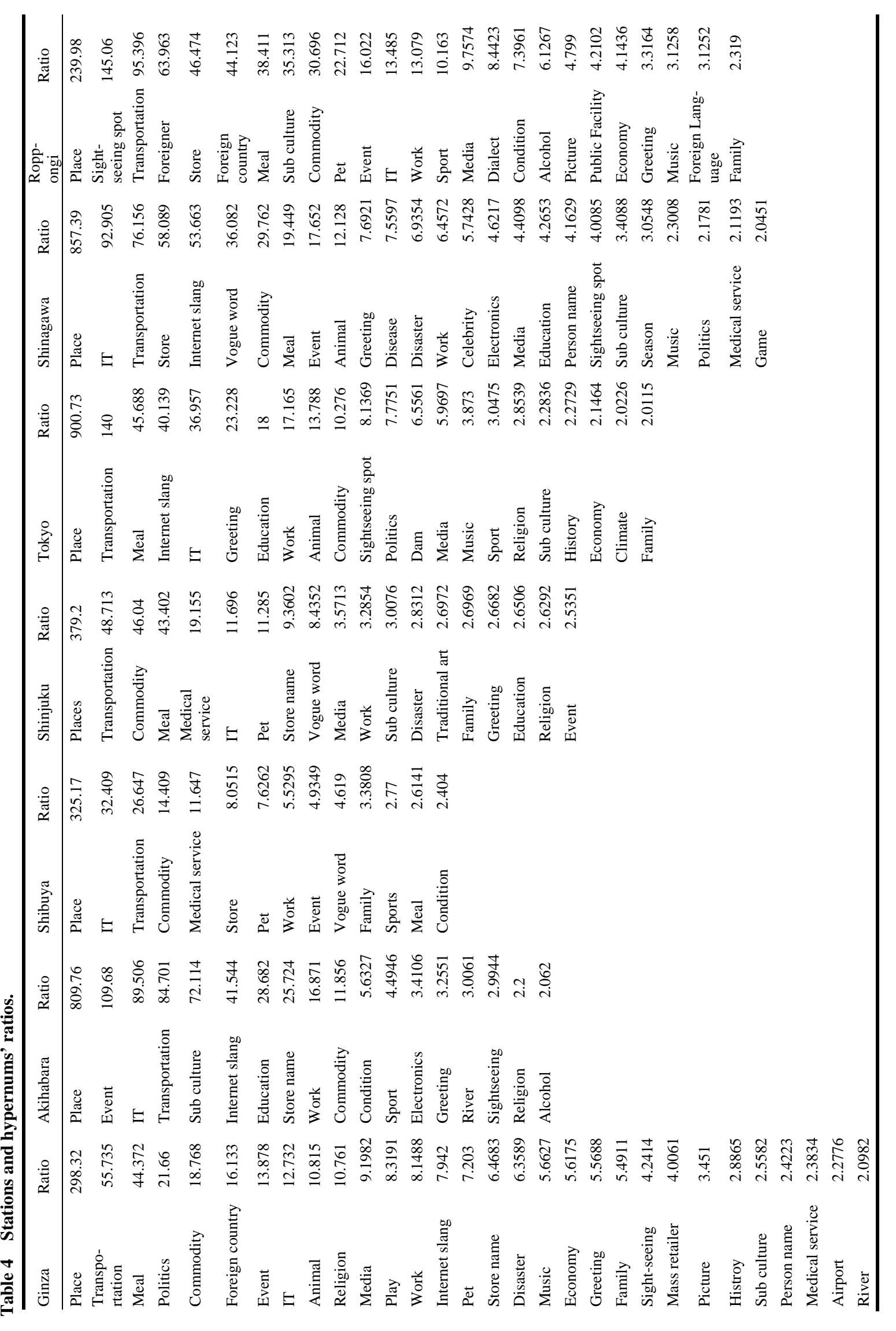


were relatively high. Lastly in Shibuya and Shinjuku, "place”, "IT”, “transportation”, “commodity” and "medical service” were common frequent hypernyms. As a result, correlation method can elucidate the similarity of each station as it like a remote sensor.

Although Roppongi belong to the kind of extreme weak correlation in Table 3, some interesting similarity with Akihabara can be discovered in Table 4 such as "Sub Culture" and "IT". Discrepancy between two methods basically came from the diversity of "words". Thanks to the colloquial language and internet slang, the authors' expression is thought to become miscellaneous. These diverse elocutions can bury the potential user's trend.

In summary, correlation method can explain the similarity between station market trends while possibilities can be discovered by using hypernym methods

\section{Conclusions}

Big data of geo-tagged user was collected by using Twitter API, and was analyzed by using Japanese morphological analysis and terminology extraction. Also, the number of user entering some market area was analyzed by using Hubeny Formula. Combining results of texts and GPS, the each station's keywords and scores were extracted. After calculating ratios between all users' scores of keywords and those in station within $400 \mathrm{~m}$, the authors tagged and sorted the keywords and scores. At analyzing station market area, correlation and hypernym method were applied.

Correlation between each station can work as remote sensor of finding similarity and difference of stations. On the other hand, hypernym method can discover the potential in market area.

Limitations aroused at methods. The authors had to generalize the meaning of each word at hypernum method without computational method. Also, the author had to select the number of keywords and ratios arbitrarily at hypernym method. Lastly, these methods have not studied the relationship between each score of keywords and numbers of store, which will be most instructive to determine the function of architecture or city.

After solving these problems, all the full-developed cities can be analyzed the characteristics. Not only architectural planning and urban planning, but also real estate development and real estate marketing will be significantly improved based on this method.

\section{Acknowledgments}

This research was supported by grant-in-aid for Challenging Exploratory Research (23656370).

\section{References}

[1] R. Nakamura, K. Yoshida, H. Watanabe, C. Izumi, Y. Ikehara, Behavioral spesifity in environment, Transactions of the Architectural Institute of Japan 180 (1971) 45-52.

[2] B. Hiller, A. Leaman, P. Stansall, M. Bedford, Space syntax, Environment and Planning B: Planning and Design 3 (2) (1960) 147-185.

[3] K. Lynch, The Image of the City, The MIT Press, Massachusetts, 1960.

[4] D. Hayden, The Power of Place: Urban Landscape as Public history, The MIT Press, Massachusetts, 1995.

[5] R. Florida, Who's Your City?: How the Creative Economy Is Making Where to Live the Most Important Decision of Your Life, Basic Book, New York, 2008.

[6] Twitter Developers: Public Stream, https://dev.twitter.com/docs/streaming-apis/streams/publi c (accessed May 17, 2012).

[7] Twitter Developers: Get Statuses/User_Timeline, https://dev.twitter.com/docs/api/1/get/statuses/user_timeli ne (accessed May 10, 2012).

[8] Japan Developer Network Website, http://developer.yahoo.co.jp/webapi/map/openlocalplatfor m/v1/static.html (accessed Aug. 18, 2012).

[9] MeCab: Yet Another Part-of-Speech and Morphological Analyzer,

http://mecab.googlecode.com/svn/trunk/mecab/doc/index. html (accessed Aug. 18, 2012).

[10] H. Nakagawa, A. Maeda, H. Kojima, TermExtract: Perl’s module for Automatic Terminology Extraction, http://gensen.dl.itc.u-tokyo.ac.jp /termextract.html (accessed Aug. 18, 2012).

[11] CodePlus: Station Database, http://www.ekidata.jp/download/index.html (accessed June 26, 2012). 